\title{
Endoscopic Ultrasound-Guided Fine-Needle Aspiration for Focal Liver Lesions: The Expanding Light Over the Shadow
}

\author{
Takuji Iwashita and Masahito Shimizu \\ First Department of Internal Medicine, Gifu University Hospital, Gifu, Japan
}

See "Endoscopic Ultrasound-Guided Fine Needle Aspiration Using a 22-G Needle for Hepatic Lesions: Single-Center Experience" by Ebru Akay, Deniz Atasoy, Engin Altınkaya et al., on page 404-412.

Endoscopic ultrasound (EUS) has enabled detailed observation of lesions around the upper intestine, as well as aided the acquisition of pathological samples using fine-needle aspiration (FNA) under real-time EUS image guidance from the within the intestine. EUS is a highly sensitive imaging technique, and EUS-FNA is a reliable and safe biopsy procedure used in daily clinical practice. ${ }^{1,2}$ Moreover, both are commonly used for evaluating pancreatic lesions since the pancreas is located in the deep abdomen, making percutaneous imaging and biopsy difficult. On the other hand, abdominal ultrasound is a well-established modality for the evaluation of liver lesions, which is followed by percutaneous biopsy when necessary; however, the indications for EUS have also recently expanded to liver lesions.

A study in 1999 by Nguyen et al. ${ }^{3}$ provided an initial report of EUS-FNA for liver lesions. They reported that EUS revealed the presence of focal liver lesions in 14 of 574 (2.4\%) patients with a history or with suspicion of malignancy, whereas previous computed tomography scans depicted the liver lesions in only 3 out of $14(21 \%)$ patients. EUS-FNA using a 22-gauge needle was subsequently performed for these liver lesions

Received: April 7, 2021 Revised: April 22, 2021

Accepted: April 22, 2021

Correspondence: Takuji Iwashita

First Department of Internal Medicine, Gifu University Hospital, 1-1 Yanagido, Gifu 501-1194, Japan

Tel: +81-58-230-6308, Fax: +81-58-230-6310, E-mail: takuji@w7.dion.ne.jp ORCID: https://orcid.org/0000-0003-4978-1787

cc This is an Open Access article distributed under the terms of the Creative Commons Attribution Non-Commercial License (http://creativecommons.org/ licenses/by-nc/3.0) which permits unrestricted non-commercial use, distribution, and reproduction in any medium, provided the original work is properly cited. (median size, $1.1 \mathrm{~cm}$; range, 0.8 to $5.2 \mathrm{~cm}$ ) with a mean number of passes of 2.0 (range, 1-5), yielding adequate samples for cytological evaluation without any adverse events. The authors then concluded that EUS and EUS-FNA established a definitive M stage. These study findings show that EUS can detect small focal liver lesions and confirm that a cytologic diagnosis of liver metastasis using FNA may impact future clinical management. Following this report, several groups have begun evaluating the efficacy and safety of EUS-FNA for focal liver lesions. A retrospective cohort study including 41 patients showed that EUS-FNA was feasible in 40 of them (97.5\%). The sensitivity and specificity for malignancy were $94 \%$ and $100 \%$, respectively, with an average needle pass of 1.4 passes, ${ }^{4}$ and minor adverse events (self-limited local bleeding) only occurred in two patients (4.8\%). Since there have been no studies comparing EUS-FNA and other modalities, no conclusion has been made regarding the superiority of EUS-FNA for liver lesions; however, based on the results of these reports, EUSFNA can be considered a feasible, reliable, and safe procedure for focal liver lesions.

Despite the excellent results of EUS-FNA for liver lesions, its indications for use remain unclear. One of the reasons for this might be the visualization capability of EUS for the liver. EUS is equipped with a high-frequency transducer that provides high-resolution images but has a limited visualization range which obscures deep liver observation. Furthermore, the liver can only be visualized from the stomach and duodenum during EUS, which results in a restricted visible area. These features of EUS only enables detailed observation of the liver's left lateral, caudate, and partial right lobes, limiting the indica- 
tion of EUS for liver lesion screening. On the other hand, EUSFNA has advantages over percutaneous biopsy. First, since the EUS-FNA access route is from within the body, it is minimally affected by ascites surrounding the liver and is completely unaffected by subcutaneous fat. Second, the deep area in the percutaneous approach can be a shallow area in EUS-FNA. An example of this situation is the liver's caudate lobe, which is located in a deep area just behind the inferior vena cava using the percutaneous approach, but this becomes an area adjacent to the stomach using the EUS approach.

In this issue of Clinical Endoscopy, a study by Akay et al. ${ }^{5}$ evaluated the diagnostic capability of a single EUS-FNA puncture using a 22-gauge needle for focal liver lesions, which included 25 patients with liver lesions and a technical success rate of $88 \%(22 / 25)$. Of the 22 patients with successful EUSFNA, the aspirate sufficiency and biopsy sufficiency rates were 94\% (21/22) and 86\% (19/22), respectively, even with a single pass of FNA. Moreover, the procedure itself had a sensitivity, specificity, and accuracy of $94 \%, 100 \%$, and $86 \%$, respectively, with no adverse post-procedure events occurring. The results of this study confirmed the efficacy and safety of EUS-FNA for treating liver lesions, and it also suggested that a single pass of FNA using a regular 22-gauge needle might be sufficient to obtain a diagnostic pathological specimen. Recently, fine-needle biopsy (FNB) needles with a Franseen or Fork-tip shape have been developed, with reports showing a high diagnostic yield with fewer needle passes. ${ }^{6,7}$ Thus, a single pass of EUSFNB may further improve the diagnostic capability of liver lesions.

Recent development of devices and deepening knowledge of EUS-related procedures have expanded EUS indications for liver disease diagnosis and treatment of liver. ${ }^{8} \mathrm{~A}$ meta-analysis of EUS-FNA for liver biopsy using a larger bore needle (19-gauge) showed a histologic diagnosis rate of $93.9 \%$ and an adverse event rate of $2.3 \%$, concluding that EUS-guided liver biopsy is an effective and safe sampling method. ${ }^{9}$ EUS-guided portal pressure gradient (PPG) measurement using a 25-gauge FNA needle and a novel compact manometer showed a 100\% technical success, no adverse events, and PPG with excellent correlation with clinical parameters. ${ }^{10}$ Other than these new indications, there have been reports of EUS-guided liver tumor treatments using thermal therapy, including radiofrequency ablation, laser ablation or cryoablation, brachytherapy, or photodynamic therapy, although most of them are still in research protocols. ${ }^{8}$ Considering these reports regarding new knowledge, techniques, and devices in EUS-guided interventions, the indication "light" of EUS-guided management for the liver will expand over the current negative side "shadow" of EUS.

Conflicts of Interest

The author has no potential conflicts of interest.

Funding

None.

ORCID

Takuji Iwashita:

Masahito Shimizu:

https://orcid.org/0000-0003-4978-1787 https://orcid.org/0000-0002-1151-2058

\section{REFERENCES}

1. Hewitt MJ, McPhail MJW, Possamai L, Dhar A, Vlavianos P, Monahan KJ. EUS-guided FNA for diagnosis of solid pancreatic neoplasms: a meta-analysis. Gastrointest Endosc 2012;75:319-331.

2. Kanno A, Yasuda I, Irisawa A, et al. Adverse events of endoscopic ultrasound-guided fine-needle aspiration for histologic diagnosis in Japanese tertiary centers: Multicenter retrospective study. Dig Endosc 2020 Dec 7 [Epub] https://doi.org/10.1111/den.13912.

3. Nguyen P, Feng JC, Chang KJ. Endoscopic ultrasound (EUS) and EUS-guided fine-needle aspiration (FNA) of liver lesions. Gastrointest Endosc 1999;50:357-361.

4. Hollerbach S, Willert J, Topalidis T, Reiser M, Schmiegel W. Endoscopic ultrasound-guided fine-needle aspiration biopsy of liver lesions: histological and cytological assessment. Endoscopy 2003;35:743-749.

5. Akay E, Atasoy D, Altınkaya E, et al. Endoscopic ultrasound-guided fine needle aspiration using a 22-G needle for hepatic lesions: single-center experience. Clin Endosc 2021;54:404-412.

6. Bang JY, Kirtane S, Krall K, et al. In memoriam: Fine-needle aspiration, birth: Fine-needle biopsy: The changing trend in endoscopic ultrasound-guided tissue acquisition. Dig Endosc 2019;31:197-202.

7. Mita N, Iwashita T, Uemura S, et al. Endoscopic ultrasound-guided fine needle biopsy using 22-gauge franseen needle for the histological diagnosis of solid lesions: a multicenter prospective pilot study. Dig Dis Sci 2020;65:1155-1163.

8. Hashimoto R, Chang KJ. Endoscopic ultrasound guided hepatic interventions. Dig Endosc 2021;33:54-65.

9. Mohan BP, Shakhatreh M, Garg R, Ponnada S, Adler DG. Efficacy and safety of EUS-guided liver biopsy: a systematic review and meta-analysis. Gastrointest Endosc 2019;89:238-246.e3.

10. Samarasena JB, Huang JY, Tsujino T, et al. EUS-guided portal pressure gradient measurement with a simple novel device: a human pilot study. VideoGIE 2018;3:361-363. 